\title{
Retracts of Products of Chordal Graphs
}

\begin{abstract}
Boštjan Brešar, ${ }^{1}$ Jérémie Chalopin, ${ }^{2}$ Victor Chepoi, ${ }^{2}$ Matjaž Kovše, ${ }^{1}$ Arnaud Labourel, ${ }^{2}$ and Yann Vaxès ${ }^{2}$

${ }^{1}$ FACULTY OF NATURAL SCIENCES AND MATHEMATICS UNIVERSITY OF MARIBOR

KOROŠKA CESTA 160, SI-2000, MARIBOR, SLOVENIA

E-mail: bostjan.bresar@uni-mb.si; matjaz.kovse@uni-mb.si

${ }^{2}$ LIF, AIX-MARSEILLE UNIVERSITÉ AND CNRS, FACULTÉ DES SCIENCES DE LUMINY MARSEILLE, FRANCE E-mail: jeremie.chalopin@lif.univ-mrs.fr; victor.chepoi@lif.univ-mrs.fr; arnaud.labourel@lif.univ-mrs.fr; yann.vaxes@lif.univ-mrs.fr
\end{abstract}

Received January 5, 2011; Revised February 12, 2012

Published online in Wiley Online Library (wileyonlinelibrary.com).

DOI 10.1002/jgt.21665

\begin{abstract}
In this article, we characterize the graphs $G$ that are the retracts of Cartesian products of chordal graphs. We show that they are exactly the weakly modular graphs that do not contain $K_{2,3}$, the 4-wheel minus one spoke $W_{4}^{-}$, and the $k$-wheels $W_{k}$ (for $k \geq 4$ ) as induced subgraphs. We also show that these graphs $G$ are exactly the cage-amalgamation graphs as introduced by Brešar and Tepeh Horvat (Cage-amalgamation graphs, a common generalization of chordal and median graphs, Eur J Combin 30 (2009), 1071-1081); this solves the open question raised by these authors. Finally, we prove that replacing all products of cliques of $G$ by products of Euclidean simplices, we obtain a polyhedral cell complex which, endowed
\end{abstract}

Contract grant sponsor: French-Slovenian Egide PROTEUS project; Contract grant sponsor: Ministry of Science and Technology of Slovenia; Contract grant numbers: J1-2043 and P1-0297 (B. B. and M. K.); Contract grant sponsor: TEOMATRO; Contract grant number: ANR-10-BLAN 0207 (V. C.).

Journal of Graph Theory

(C) 2012 Wiley Periodicals, Inc. 
with an intrinsic Euclidean metric, is a CAT(0) space. This generalizes similar results about median graphs as retracts of hypercubes (products of edges) and median graphs as 1-skeletons of CAT(0) cubical complexes. (c) 2012 Wiley Periodicals, Inc. J. Graph Theory XX: 1-20, 2012

Keywords: median graph; chordal graph; retract; Cartesian product; CAT(0) cubical complexes

\section{INTRODUCTION}

Median graphs constitute one of the most important classes of graphs investigated in metric graph theory and occur in different areas of discrete mathematics, metric geometry, and computer science. Median graphs and related median structures (median algebras and median complexes) have many nice properties and admit numerous characterizations. All median structures are intimately related to hypercubes: median graphs are isometric subgraphs of hypercubes; in fact, by a classical result of Bandelt [1], they are the retracts of hypercubes into which they embed isometrically. It was also shown by Isbell [25] and van de Vel [33] that every finite median graph $G$ can be obtained by successive applications of gated amalgamations from hypercubes, thus showing that the only prime median graph is the two-vertex complete graph $K_{2}$ (a graph with at least two vertices is said to be prime if it is neither a Cartesian product nor a gated amalgam of smaller graphs). A related construction of median graphs via convex expansions is given in [27, 28]. Median graphs also have a remarkable algebraic structure, which is induced by the ternary operation on the vertex set that assigns to each triplet of vertices the unique median vertex, and their algebra can be characterized using four natural axioms [7, 25] among all discrete ternary algebras. Finally, it was shown in [16], [29] that the cubical complexes derived from median graphs by replacing graphic cubes by solid cubes are exactly the CAT(0) cubical complexes. Thus, due to a result of Gromov [22], median complexes can be characterized as simply connected cubical complexes with triangle-free links of vertices. For more detailed information about median structures, the interested reader can consult the survey [6] and the books [19, 24, 28, 34].

This structure theory of graphs based on two fundamental operations, viz., Cartesian multiplication and gated amalgamation, was further elaborated for more general classes of graphs. Some of the results for median graphs have been extended to quasi-median graphs introduced by Mulder [28] and further studied in [8, 10, 35]: quasi-median graphs are precisely the weakly modular graphs not containing induced $K_{2,3}$ and $K_{4}-e$; they can also be characterized as the retracts of Hamming graphs (Cartesian products of complete graphs) and can be obtained from complete graphs by Cartesian products and gated amalgamations. More recently, Bandelt and Chepoi [3]-[5] presented a similar decomposition scheme of weakly median graphs and characterized the prime graphs with respect to this decomposition: the hyperoctahedra and their subgraphs, the 5-wheel $W_{5}$, and the 2-connected plane triangulations in which all inner vertices have degrees $\geq 6$. Using these results and a result of Chastand $[13,14]$, they further showed that weakly median graphs are the retracts of the Cartesian products of their primes and presented an axiomatic characterization of underlying weakly median algebras. The extensive research on generalizations of median graphs leads to a general framework for the study of classes 
of graphs, closed for Cartesian products and gated amalgamations, proposed in [9, 13, 14].

In this article, we continue this line of research and characterize the graphs $G$ which are retracts of Cartesian products of chordal graphs. We show that they are exactly the weakly modular graphs which do not contain $K_{2,3}, W_{4}^{-}$and the $k$-wheels $W_{k}$ for $k \geq 4$ as induced subgraphs. We establish that these graphs $G$ are exactly the cage-amalgamation graphs as introduced by Brešar and Tepeh Horvat [11], that is, the graphs which can be obtained via successive gated amalgamations from Cartesian products of chordal graphs; this solves the open question raised in [11]. This result along with definitions and preliminary observations is presented in the next section, while its proof is the contents of Section 3. Finally in Section 4, we show that replacing all products of cliques of $G$ by products of Euclidean simplices, we will obtain a polyhedral cell complex which, endowed with an intrinsic $l_{2}$-metric, is a CAT(0) space. This generalizes similar results about median graphs as retracts of hypercubes (products of edges) and median graphs as 1-skeletons of $\mathrm{CAT}(0)$ cubical complexes.

\section{PRELIMINARIES AND THE CHARACTERIZATIONS}

In this section, we present all the necessary definitions and preliminary results so that at the end we formulate two characterizations of the retracts of products of chordal graphs.

All graphs $G=(V, E)$ occurring in this article are undirected, connected, and without loops or multiple edges. The distance $d(u, v)$ between two vertices $u$ and $v$ is the length of a shortest $(u, v)$-path, and the interval $I(u, v)$ between $u$ and $v$ consists of all vertices on shortest $(u, v)$-paths, that is, of all vertices (metrically) between $u$ and $v: I(u, v)=\{x \in$ $V: d(u, x)+d(x, v)=d(u, v)\}$. An induced subgraph of $G$ (or the corresponding vertex set $A$ ) is called convex if it includes the interval of $G$ between any pair of its vertices. An induced subgraph $H$ of a graph $G$ is said to be gated if for every vertex $x$ outside $H$ there exists a vertex $x^{\prime}$ (the gate of $x$ ) in $H$ such that each vertex $y$ of $H$ is connected with $x$ by a shortest path passing through the gate $x^{\prime}$ (i.e., $x^{\prime} \in I(x, y)$ ). The smallest convex (or gated, respectively) subgraph containing a given subgraph $S$ is the convex hull (or gated hull, respectively) of $S$. A graph $G=(V, E)$ is isometrically embeddable into a graph $H=(W, F)$ if there exists a mapping $\varphi: V \rightarrow W$ such that $d_{H}(\varphi(u), \varphi(v))=d_{G}(u, v)$ for all vertices $u, v \in V$. A retraction $\varphi$ of $H$ is an idempotent nonexpansive mapping of $H$ into itself, that is, $\varphi^{2}=\varphi: W \rightarrow W$ with $d(\varphi(x), \varphi(y)) \leq d(x, y)$ for all $x, y \in W$. The subgraph of $H$ induced by the image of $H$ under $\varphi$ is referred to as a retract of $H$.

A graph $G$ is a gated amalgam of two graphs $G_{1}$ and $G_{2}$ if $G_{1}$ and $G_{2}$ are (isomorphic to) two intersecting gated subgraphs of $G$ whose union is all of $G$. The Cartesian product [24] $G=G_{1} \square \cdots \square G_{n}$ of $n$ graphs $G_{1}, \ldots, G_{n}$ has the $n$-tuples $\left(x_{1}, \ldots, x_{n}\right)$ as its vertices (with vertex $x_{i}$ from $\left.G_{i}\right)$ and an edge between two vertices $x=\left(x_{1}, \ldots, x_{n}\right)$ and $y=\left(y_{1}, \ldots, y_{n}\right)$ if and only if, for some $i$, the vertices $x_{i}$ and $y_{i}$ are adjacent in $G_{i}$, and $x_{j}=y_{j}$ for the remaining $j \neq i$. Obviously, $d_{G}(u, v)=\sum_{i=1}^{n} d_{G_{i}}\left(u_{i}, v_{i}\right)$ for any two vertices $u=\left(u_{1}, \ldots, u_{n}\right)$ and $v=\left(v_{1}, \ldots, v_{n}\right)$ of $G$. In regard to a decomposition scheme involving multiplication and amalgamation, a graph with at least two vertices is said to be prime if it is neither a Cartesian product nor a gated amalgam of smaller graphs. For instance, the only prime median graph is the two-vertex complete graph $K_{2}$ $[25,33]$ and the prime quasi-median graphs are exactly the complete graphs $[8,25]$. 

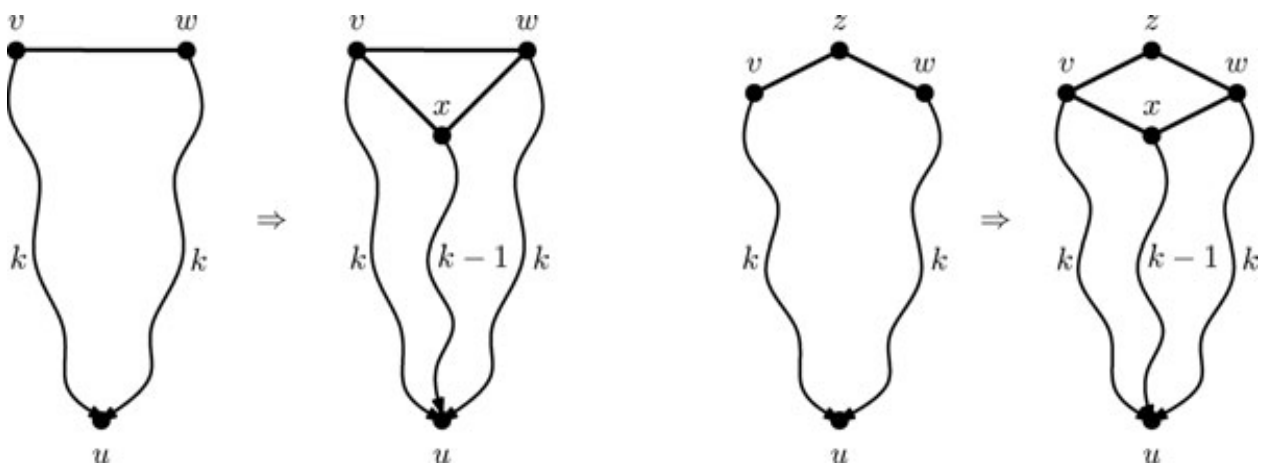

FIGURE 1. Triangle and quadrangle conditions.

A graph $G$ is weakly modular $[2,15]$ if its distance function $d$ satisfies the following triangle and quadrangle conditions (see Fig. 1):

Triangle condition: For any three vertices $u, v, w$ with $1=d(v, w)<d(u, v)=d(u, w)$ there exists a common neighbor $x$ of $v$ and $w$ such that $d(u, x)=d(u, v)-1$.

Quadrangle condition: For any four vertices $u, v, w, z$ with $d(v, z)=d(w, z)=1$ and $2=d(v, w) \leq d(u, v)=d(u, w)=d(u, z)-1$, there exists a common neighbor $x$ of $v$ and $w$ such that $d(u, x)=d(u, v)-1$.

A weakly median graph is a weakly modular graph in which the vertex $x$ defined in the triangle and quadrangle conditions is always unique. Equivalently, weakly median graphs can be defined as the weakly modular graphs in which each triplet of vertices has a unique quasi-median. Median graphs are the bipartite weakly median graphs and, equivalently, can be defined as the graphs in which each triplet of vertices $u, v, w$ has a unique median vertex, that is, $|I(u, v) \cap I(u, w) \cap I(v, w)|=1$. Bridged graphs constitute another important subclass of weakly modular graphs. Recall that a graph is called bridged $[17,18,31]$ if it does not contain any isometric cycle of length greater than 3 , or alternatively, if the closed neighborhood $N[A]=A \cup\{y \in V: y$ is adjacent to some $x \in$ $A$ ) of every convex set $A$ of $G$ is convex. Chordal graphs constitute the most famous subclass of bridged graphs. A graph is said to be chordal if it does not contain induced cycles of length greater than 3 . In this article, we will investigate the finite graphs $G$, which are obtained from Cartesian products of chordal graphs via gated amalgamations. These graphs have been introduced by Brešar and Tepeh Horvat [11] and are called cageamalgamation graphs. More precisely, the Cartesian products of connected cutvertexfree chordal graphs were called in [11] cages, and the graphs that can be obtained by a sequence of gated amalgamations from cages were called cage-amalgamation graphs. It can be easily shown that cage-amalgamation graphs are weakly modular graphs and that they do not contain induced $K_{2,3}$, wheels $W_{k}$, and almost-wheels $W_{k}^{-}$(the wheel $W_{k}$ is a graph obtained by connecting a single vertex - the central vertex - to all vertices of the $k$-cycle; the almost-wheel $W_{k}^{-}$is the graph obtained from $W_{k}$ by deleting a spoke (i.e., an edge between the central vertex and a vertex of the $k$-cycle), see Figure 2 for examples. It was conjectured in [11] that in fact this list of forbidden subgraphs completely characterizes the cage-amalgamation graphs. The main result of our article proves this conjecture (in fact, we note that just $W_{4}^{-}$suffices in the list of forbidden almost wheels). 


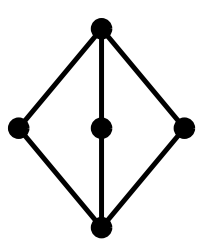

$K_{2,3}$

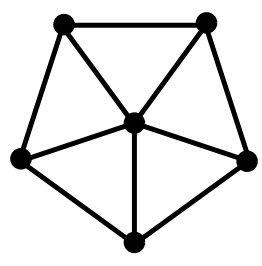

$W_{5}$

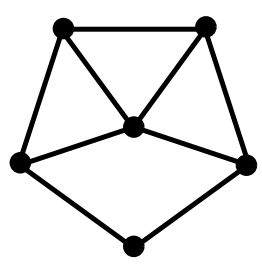

$W_{5}^{-}$

FIGURE 2. The complete bipartite graph $K_{2,3}$, the wheel $W_{5}$, and the almost-wheel $W_{5}^{-}$.

Theorem 1. For a finite graph $G=(V, E)$, the following conditions are equivalent:

(i) $G$ is a retract of the Cartesian product of chordal graphs.

(ii) $G$ is a weakly modular graph not containing induced $K_{2,3}, W_{4}^{-}$and wheels $W_{k}$, for $k \geq 4$.

(iii) $G$ is a cage-amalgamation graph, that is, it can be obtained by successive applications of gated amalgamations from Cartesian products of 2-connected chordal graphs and $K_{2}$ 's.

The proof of this theorem is provided in the following section. The most difficult part of the proof is the implication (ii) $\Rightarrow$ (iii), which we establish in two steps. First, we show that if $G$ is a weakly modular graph not containing induced $K_{2,3}$, wheels $W_{k}$, and almost-wheels $W_{k}^{-}$for $k \geq 3$, then all its prime graphs are 2-connected chordal graphs or a $K_{2}$. In the second part, using the techniques developed in [3], we show that $G$ can be obtained via gated amalgamations from Cartesian products of prime graphs.

\section{PROOF OF THEOREM 1}

The implication (i) $\Rightarrow$ (ii) is obvious: chordal graphs are weakly modular and do not contain induced $K_{2,3}$, wheels $W_{k}$, and almost-wheels $W_{k}^{-}(k \geq 4)$. Weakly modular graphs are closed by taking Cartesian products. If a Cartesian product of $k$ graphs $H_{1}, \ldots, H_{k}$ contains an induced $K_{2,3}, W_{k}$, or $W_{k}^{-}$, then necessarily this graph occurs in one of the factors $H_{i}$. As a consequence, Cartesian products $H=H_{1} \square \cdots \square H_{k}$ of chordal graphs do not contain induced $K_{2,3}, W_{k}$, and $W_{k}^{-}$. If $G$ is a retract of $H=H_{1} \square \cdots \square H_{k}$, then $G$ is an isometric subgraph of $H$ and therefore $G$ does not contain induced $K_{2,3}, W_{k}$, and $W_{k}^{-}$either. It remains to notice that triangle and quadrangle conditions are preserved by Cartesian products and retractions, thus $G$ is a weakly modular graph, establishing that (i) $\Rightarrow$ (ii).

The implication (iii) $\Rightarrow$ (i) is a particular case of Theorem 1 and Corollary 4 of [4] (the proof of Corollary 4 also follows from a more general result of Chastand [14]). By Theorem 1 of [4], any cage-amalgamation graph $G$ embeds isometrically into the Cartesian product $H=H_{1} \square \cdots \square H_{k}$ of its prime graphs. Corollary 4 of [4] then shows that there exists a retraction from $H$ to $G$, establishing (iii) $\Rightarrow$ (i).

The proof of the implication (ii) $\Rightarrow$ (iii) is the main contribution of this section. We start with the lemma that shows that only $W_{4}^{-}$suffices in the list of forbidden almost wheels. 
Lemma 1. Let $G$ be a weakly modular graph without induced $W_{4}$ and $W_{4}^{-}$. Then $G$ does not contain an induced $W_{n}^{-}$for $n>4$.

Proof. Suppose by way of contradiction that $W_{n}^{-}$is an induced subgraph of $G$ and suppose that $G$ does not contain any induced $W_{k}^{-}$for any $3<k<n$. Let $\left(x_{1}, x_{2}, \ldots, x_{n}, x_{1}\right)$ be the outer cycle $C$ of $W_{n}^{-}$and consider a vertex $c$ adjacent to all vertices of $C$ except $x_{1}$. We apply the triangle condition to the triple $x_{1}, x_{2}, x_{n-1}$ and find a vertex $a \in N\left(x_{1}\right) \cap N\left(x_{2}\right) \cap N\left(x_{n-1}\right)$. Note that if $a \sim c$, then $x_{1}, x_{2}, c, x_{n}, a$ induce a $W_{4}$ if $a$ is adjacent to $x_{n}$ or a $W_{4}^{-}$otherwise. Assume now that $a \neq c$. If $n=5$, then the vertices $x_{4}, a, x_{2}, c, x_{3}$ induce either a $W_{4}$ if $x_{3}$ is adjacent to $a$, or a $W_{4}^{-}$otherwise. Now, if $n \geq 6$, the subgraph induced by the vertices $a, x_{2}, x_{3}, \ldots, x_{n-1}, c$ is isomorphic to one of the forbidden induced subgraphs $W_{k}^{-}$, where $k<n$, unless $a$ is adjacent to $x_{3}, x_{4}, \ldots, x_{n-2}$. Thus, $a$ is adjacent to all vertices of $C$ except maybe $x_{n}$. The vertices $a, x_{3}, c, x_{n-1}, x_{4}$ induce a $W_{4}$, if $n=6$, or a $W_{4}^{-}$otherwise, a contradiction.

The proof of (ii) $\Rightarrow$ (iii) employs the fact that each finite chordal graph admits a perfect elimination scheme, which can be computed by maximum cardinality search (MCS) algorithm [20, 30, 32]. Running a modification of MCS on the gated hull of a triangle in a graph $G$ satisfying the condition (ii) of Theorem 1, we show that the level subgraphs returned by MCS are all convex subgraphs of $G$. This allows us to show that the gated hull of each triangle of $G$ is a 2-connected chordal graph, thus identifying the prime graphs of $G$. To show that $G$ can be obtained from Cartesian products of 2-connected chordal graphs and edges using successive amalgamations, we adapt the second part of the proof of Theorem 1 of [3].

A simplicial vertex of a graph $G$ is a vertex $v$ such that its neighborhood $N(v)=$ $\{u \in V(G): u$ is adjacent to $v\}$ induces a complete subgraph of $G$. A perfect elimination ordering (PEO) of a graph $G=(V, E)$ with $n$ vertices is a total ordering $v_{1}, \ldots, v_{n}$ of its vertices such that each $v_{i}$ is a simplicial vertex in the subgraph $G_{i}$ induced by the level set $L_{i}=\left\{v_{1}, \ldots, v_{i}\right\}$. It is well known (see [20]) that a finite graph $G$ admits a PEO if and only if $G$ is chordal. A PEO of a chordal graph $G$ can be found (in linear time) either using Lexicographic Breadth-First-Search (LexBFS) [30] or MCS introduced by Tarjan and Yannakakis [32]. MCS algorithm works as follows: the first vertex is chosen arbitrarily, and the $(i+1)$ th vertex is the unlabeled vertex that has the largest number of already numbered neighbors, breaking ties arbitrarily. We will denote by $\alpha(v)$ the number of $v$ in a total ordering $v_{1}, \ldots, v_{n}$, that is, if $\alpha(v)=i$, then $v=v_{i}$. We start with two properties of MCS in chordal graphs.

The following result is a part of folklore, and we give its short proof only to make the article self-contained.

Lemma 2. Let $G$ be a chordal graph and $\alpha$ an ordering of vertices produced by MCS. If a vertex $z$ belongs to an induced path between two vertices $x, y$, then $\alpha(z)<$ $\max \{\alpha(x), \alpha(y)\}$.

Proof. Assume without loss of generality that $\alpha(x)<\alpha(y)$ and let $P$ be an induced path between $x$ and $y$. Suppose by way of contradiction that $P$ contains a vertex $z$ such that $\alpha(z)>\alpha(y)$ and suppose without loss of generality that $z$ is the vertex of $P$ with the largest index $i=\alpha(z)$. Then among all vertices of $P$ the vertex $z$ was labeled last. Hence $z$ and its neighbors $z^{\prime}, z^{\prime \prime}$ in $P$ all belong to the subgraph $G_{i}$. Since $z^{\prime}$ and $z^{\prime \prime}$ are not 
adjacent, $z$ is not a simplicial vertex of $G_{i}$, contradicting the fact that on chordal graphs MCS returns a PEO.

A minimal (vertex) separator of a graph $G=(V, E)$ is a subset of vertices $K$ of $G$ such that the subgraph of $G$ induced by $V-K$ contains at least two connected components $A$ and $B$, and that $K$ is minimal by inclusion with respect to this separating property. Then $K$ necessarily separates any two vertices $x \in A$ and $y \in B$ in the sense that all $(x, y)$-paths share a vertex with $K$. It is well known [20] that any minimal separator $K$ of a chordal graph $G$ induces a complete subgraph of $G$ and, moreover, $K$ separates two vertices $x$ and $y$ such that both $x$ and $y$ are adjacent to all vertices of $K$.

Lemma 3. Let $K$ be a minimal separator of a chordal graph $G$, let $A$ and $B$ be two connected components of $G-K$, and let $u \in A$ be a vertex that is adjacent to all vertices of $K$. Let $\alpha$ be an ordering of vertices produced by MCS. If $\alpha$ labels some vertex of $A$ before any vertex of $B$ is labeled, then $\alpha$ labels $u$ before any vertex of $B$.

Proof. Let $a_{0} \in A$ be the vertex with the smallest index $\alpha\left(a_{0}\right)$ among all vertices of $A \cup B$. Since $A$ is connected, we can choose $P:=\left(a_{0}, a_{1}, \ldots, a_{k}=u\right)$ to be a shortest (and therefore induced) path connecting the vertices $a_{0}$ and $u$ in $A$. Suppose by the way of contradiction that there exists $b \in B$ that was labeled before $u$, that is $\alpha(b)<\alpha(u)$, and let $b$ be the first such vertex with respect to $\alpha$. Denote by $L(x)$ the set of labeled neighbors of a vertex $x$ at the moment of time when $b$ was labeled. Let $K_{0}:=L(b)$. Since $K$ separates $A$ from $b \in B$, from the choice of $b$ and our assumption we conclude that $K_{0} \subseteq K$ (see also Fig. 3).

We assert that for each vertex $a_{i}$ of $P$, the inequality $\alpha\left(a_{i}\right)<\alpha(b)$ holds. Indeed, let $t$ be an arbitrary vertex in $K_{0}$ and let $P_{t}:=\left(a_{0}, \ldots, a_{k}, t\right)$ be the path from $a_{0}$ to $t$, obtained from $P$ by adding $t$ at the end. Since $\alpha(u)>\alpha(b)>\alpha(t)$ by the assumption and $\alpha(u)>\alpha\left(a_{0}\right)$ from the choice of $a_{0}$, by Lemma 2 the path $P_{t}$ is not induced. Since $P$ is induced, the only possible chords on this path are the chords of the form $t a_{i}$, where $0 \leq i<k$. Let $i_{t}$ be the smallest index such that $t$ and $a_{i_{t}}$ are adjacent. To avoid induced cycles of length greater than 3 in $G$, for all $j$ comprised between $i_{t}+1$ and $k$, the vertices $t$ and $a_{j}$ must be adjacent as well. Since the subpath $\left(a_{0}, \ldots, a_{i_{t}}, t\right)$ of $P_{t}$ is induced, by Lemma 2 we infer that all vertices of this path must be labeled either before $t$ or before $a_{0}$, but in either case we have $\alpha\left(a_{j}\right)<\alpha(b)$ for all $0 \leq j \leq i_{t}$ because $\alpha(b)>\max \left\{\alpha(t), \alpha\left(a_{0}\right)\right\}$. Set $q=\max \left\{i_{t}: t \in K_{0}\right\}$. As a result, we obtain the following property for the vertices of $P$ : every vertex $a_{j} \in\left\{a_{0}, \ldots, a_{q}\right\}$ was labeled before $b$, that is, $\alpha\left(a_{j}\right)<\alpha(b)$. On the other hand, all vertices $a_{q+1}, \ldots, a_{k}=u$ are adjacent to all

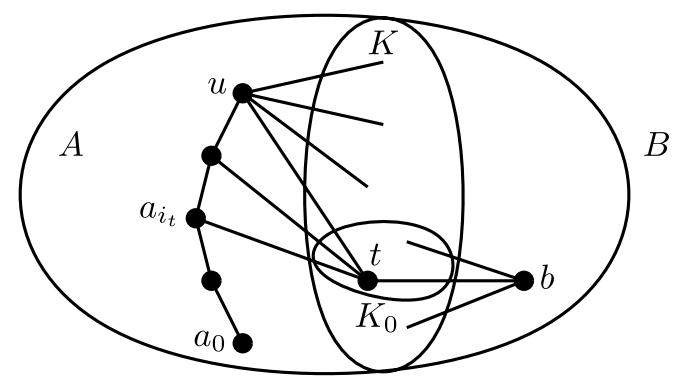

FIGURE 3. Illustration of the proof of Lemma 3. 
vertices of $K_{0}$, that is, $K_{0} \subseteq \cap_{j=q+1}^{k} L\left(a_{j}\right)$. We assert that the inclusions $K_{0} \subseteq L\left(a_{j}\right)$, $j=q+1, \ldots, k$, are strict. Since $a_{q} \in L\left(a_{q+1}\right)$, this inclusion is indeed strict for $a_{q+1}$. Let $\ell>q+1$ be the smallest index for which $L\left(a_{\ell}\right)=K_{0}$. Then, as $L(b)=K_{0}$ is a proper subset of $L\left(a_{\ell-1}\right)$, MCS must label $a_{\ell-1}$ before $b$, that is, $\alpha\left(a_{\ell-1}\right)<\alpha(b)$. Hence $a_{\ell-1} \in L\left(a_{\ell}\right)$, a contradiction. This implies, in particular, that the vertices $a_{q+1}, \ldots, a_{k}$ have been all labeled by MCS before $b$, that is, $\alpha\left(a_{j}\right)<\alpha(b)$ for $q<j \leq k$. The claimed assertion is thus proven. Now, since $a_{k}=u$, this assertion implies that $\alpha(u)<\alpha(b)$, as desired.

For the remainder of this section, let $G$ be a weakly modular graph that does not contain any of $K_{2,3}, W_{k}$, and $W_{k}^{-}, k \geq 4$, as an induced subgraph. We will show that $G$ can be obtained by a sequence of gated amalgamations from Cartesian products of chordal graphs. We commence by establishing a number of auxiliary results. A subgraph $H$ of $G$ is said to be $\Delta$-closed if, for every triangle having two vertices in $H$, the third vertex belongs to $H$ as well; then the smallest $\Delta$-closed subgraph containing $S$ is the $\Delta$-closure of $S$. In order to check whether a given subgraph of $G$ is convex or gated the following lemma is useful. This essentially coincides with Theorem 7 of [15] and can be proved quite easily by induction.

Lemma 4. A connected subgraph $H$ of a weakly modular graph $G$ is convex if and only if $H$ is locally convex, that is, for every pair of nonadjacent vertices $u, v$ of $H$ all common neighbors of $u$ and $v$ belong to $H$ whenever at least one common neighbor does. Moreover, a convex subgraph is gated if and only if it is $\Delta$-closed.

Now we will prove that the gated hull $H$ of each triangle $T=\{a, b, c\}$ of $G$ is a convex chordal subgraph of $G$. For this, we perform a (partial) MCS $\alpha$ in $G$ starting with $\alpha(a)=1, \alpha(b)=2, \alpha(c)=3$ until the moment when all yet unlabeled vertices have at most one previously labeled neighbor. Denote by $H$ the subgraph of $G$ induced by all labeled vertices at the end of the procedure, and let $H_{i}$ be the subgraph of $H$ induced by the first $i$ labeled vertices.

Proposition 1. For any $i, H_{i}$ is a chordal and convex subgraph of $G$.

Proof. We proceed by induction on $i$. Clearly, $H_{1}, H_{2}$, and $H_{3}$ are all chordal and convex subgraphs of $G$. By way of contradiction, assume that for some $i \geq 3, H_{i}$ is convex and chordal but $H_{i+1}=H_{i} \cup\left\{v_{i+1}\right\}$ is not convex. By Lemma $4, H_{i+1}$ is not locally convex. Then there exists $u \in V\left(H_{i}\right)$ such that $d_{H_{i+1}}\left(u, v_{i+1}\right)=d_{G}\left(u, v_{i+1}\right)=2$ and two vertices $x \in V\left(H_{i}\right), v \notin V\left(H_{i}\right)$, which are both adjacent to $u$ and $v_{i+1}$. Now, we will prove that any vertex in $H_{i}$, adjacent to $v_{i+1}$ is also adjacent to $v$.

Claim 1. $N\left(v_{i+1}\right) \cap H_{i} \subseteq N(v)$.

Proof of Claim 1. Let $y \in H_{i}$ be any neighbor of $v_{i+1}$ in $H_{i}$ different from $x$. From the definition of the labeling $\alpha$, we know that such a vertex exists. By induction assumption, $H_{i}$ is convex, hence $x$ and $y$ are adjacent because they have a common neighbor $v_{i+1}$ not in $H_{i}$. First, suppose that the vertices $u$ and $y$ are adjacent. To avoid forbidden $W_{4}^{-}$and $W_{4}$, the vertex $v$ must be adjacent to $x$ and to $y$, and we are done. Thus, we may assume that $u$ and $y$ are not adjacent. We distinguish two cases.

Case 1. $v$ and $x$ are not adjacent.

If $v$ and $y$ are adjacent, then we obtain a forbidden induced $W_{4}^{-}$. Thus, we may further assume that the vertices $v$ and $y$ are not adjacent (see Fig. 4, left). By the triangle condition, there exists a common neighbor $t$ of $u, v$, and $y$. Since $H_{i}$ is convex and 

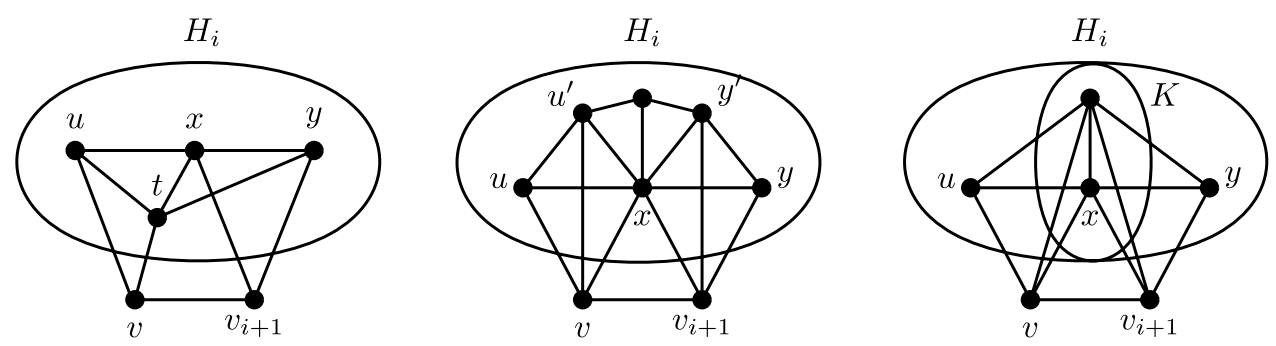

FIGURE 4. Different cases in the proof of Claim 1.

$t \in I(u, y)$, necessarily $t \in V\left(H_{i}\right)$. To avoid an induced $C_{4}$ in $H_{i}$ (which is chordal by the induction hypothesis) formed by vertices $u, t, y, x$, the vertex $t$ must be adjacent to $x$ since $u$ is not adjacent to $y$. But this leads to a contradiction, since, as $v$ is not adjacent to $x$ and $v_{i+1}$ is not adjacent to $u$, the vertices $u, v, v_{i+1}, x, t$ induce a $W_{4}^{-}$or a $W_{4}$.

Case 2. $v$ and $x$ are adjacent.

By construction, the graph $H_{i}$ is 2-connected, thus the vertices $u$ and $y$ can be connected in $H_{i}$ by an induced path $P$ that avoids $x$. Since $H_{i}$ is chordal and the path $P$ is induced, to avoid an induced cycle of length $\geq 4$ formed by some vertices of $P \cup\{x\}$, the vertex $x$ must be adjacent to all vertices of $P$. To avoid a forbidden wheel $W_{k}$ induced by $v, v_{i+1}, x$ and the vertices of $P$, necessarily $v$ or $v_{i+1}$ is adjacent to some vertex of $P$. Since $P$ is induced and $H_{i}$ is convex, $v$ can be adjacent only with the neighbor $u^{\prime}$ of $u$ in $P$ and $v_{i+1}$ can be adjacent only with the neighbor $y^{\prime}$ of $y$ in $P$. If $u^{\prime} \neq y^{\prime}$ (see Fig. 4, center) or only one of the edges $v u^{\prime}$ or $v_{i+1} y^{\prime}$ exists, then still we can find there an induced wheel $W_{k}, k \geq 4$. Hence, $u^{\prime}=y^{\prime}$ and $v, v_{i+1}$ are both adjacent to $u^{\prime}=y^{\prime}$ (see Fig. 4, right). Since the induced path $P$ is arbitrary, we infer that each induced path in $H_{i}$ between $u$ and $y$ is of length 2, and all common neighbors of $u$ and $y$ are adjacent to both $v$ and $v_{i+1}$. As a conclusion, the set $K=\left\{z \in H_{i}: u, y \in N(z)\right\}$ is a minimal (by inclusion) $(u, y)$-separator of the chordal graph $H_{i}$, and thus is a clique. Both vertices $v$ and $v_{i+1}$ are adjacent to all vertices of $K$. Let $A$ be the connected component of $H_{i}-K$ containing $u$, and let $B$ be the connected component of $H_{i}-K$ containing $y$. Suppose that the first vertex of $A \cup B$ labeled by $\alpha$ belongs to $A$. By Lemma 3, $u$ was labeled before any vertex of $B$. Let $b$ be the first vertex labeled by $\alpha$ in $B$. Let $L(x)$ denote the set of labeled vertices at the moment of time when $b$ is labeled. Then $L(b) \subseteq K$. Since, $K \cup\{u\} \subseteq L(v)$, we obtain a contradiction with the choice of MCS to label $b$ before $v$. By symmetry of $v$ and $v_{i+1}$, a similar contradiction is obtained when the first vertex of $A \cup B$ labeled by $\alpha$ belongs to $B$. This concludes the proof of the claim.

Now, Claim 1 yields $N\left(v_{i+1}\right) \cap H_{i} \subseteq N(v) \cap H_{i}$. Since $u \in H_{i}$ is adjacent to $v$ but not to $v_{i+1}$, we obtain a contradiction with the fact that MCS labels $v_{i+1}$ before $v$. Hence $H_{i+1}$ is locally convex and, therefore, a convex subgraph of $G$. It is easy to see that $H_{i+1}$ is also chordal. Indeed, since $H_{i}$ is convex, the neighborhood of $v_{i+1}$ in $H_{i}$ induces a complete subgraph, thus $v_{i+1}$ is a simplicial vertex of $H_{i+1}$. On the other hand, by the induction assumption $H_{i}$ is chordal and therefore the ordering $v_{1}, \ldots, v_{i}$ returned by MCS is a PEO of $H_{i}$. As a consequence, $v_{1}, \ldots, v_{i}, v_{i+1}$ is a PEO of $H_{i+1}$, when $H_{i+1}$ is chordal.

Proposition 2. The gated hull of $T=\{a, b, c\}$ in $G$ is the chordal subgraph $H$.

Proof. From Proposition 1 and the definition of $H$ ( $H$ is the last of the subgraphs $H_{i}$ ) we infer that $H$ is a chordal convex subgraph of $G$. $H$ is $\Delta$-closed because every vertex 


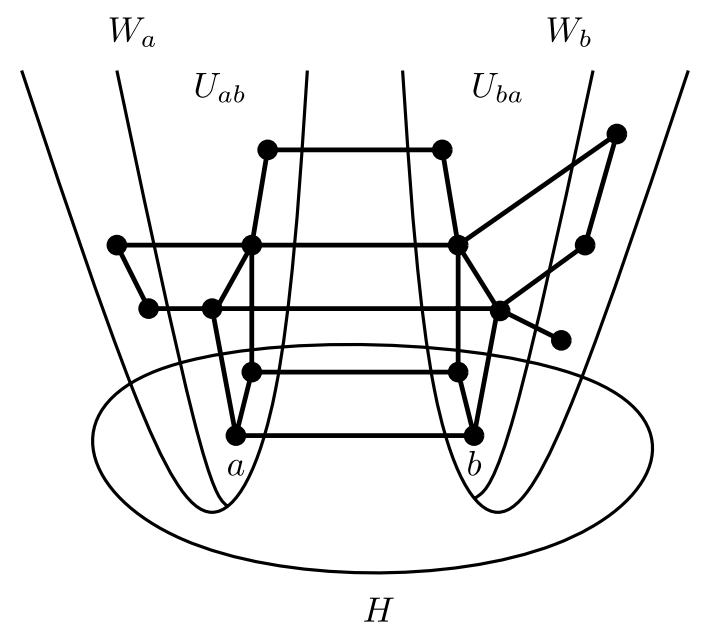

FIGURE 5. The fibers $W_{a}, W_{b}$ of the vertices $a, b \in V(H)$.

in $G-H$ has at most one neighbor in $H$, and, since $H$ is convex, by Lemma $4, H$ is a gated subgraph of $G$. On the other hand, if $H=H_{k}$, then for any index $i \leq k$, the vertex $v_{i}$ has at least two neighbors in $H_{i-1}$, thus $v_{i}$ belongs to the gated hull of $H_{i-1}$. Now, if by induction assumption $H_{i-1}$ is included in the gated hull of the triangle $T=\{a, b, c\}$, then $v_{i}$ belongs to this gated hull as well, when $H_{i}$ is contained in the gated hull of $T$, establishing the induction assertion. This shows that $H$ is contained in the gated hull of $T$. Hence, $H$ is indeed the gated hull of $T$.

Let $u v$ be an edge in $G$ and, from now on, let $H$ be the gated hull of the graph induced by $\{u, v\}$ in $G$. If $u v$ does not belong to a triangle of $G$, then $\{u, v\}$ is convex and $\Delta$-closed, thus $\{u, v\}$ itself is a gated set of $G$. In this case, $H$ is isomorphic to $K_{2}$ and is clearly chordal. If $u, v$ lie in a triangle $T$, then $H$ coincides with the gated hull of $T$ and can obtained by the (partial) MCS procedure as described above. By Proposition 2, $H$ is chordal as well.

Any gated subset $S$ of $G$ gives rise to a partition $W_{a}(a \in S)$ of the vertex set of $G$; viz., the fiber $W_{a}$ of $a$ relative to $S$ consists of all vertices $x$ (including $a$ itself) having $a$ as their gate in $S$. For adjacent vertices $a, b$ of $S$, let $U_{a b}$ be the set of vertices from $W_{a}$ which are adjacent to vertices from $W_{b}$. Let also $U_{a}=\left\{x \in W_{a}: \exists y \notin W_{a}, x y \in E(G)\right\}$. By some abuse of notation, $W_{a}, U_{a}$, and $U_{a b}$ will denote both the sets and the subgraphs induced by these sets. An example is given in Figure 5 .

Lemma 5. Each fiber $W_{a}$ relative to $H$ is gated. There exists an edge between two distinct fibers $W_{a}$ and $W_{b}$ if and only if $a$ and $b$ are adjacent.

Proof. To show that $W_{a}$ is gated, since $W_{a}$ is connected because $I(u, a) \subset W_{a}$ for any $u \in W_{a}$, by Lemma 4 , it suffices to prove that $W_{a}$ is locally convex and $\Delta$-closed. Let $x, y \in W_{a}$ have a common neighbor $z$, and, for the purposes of contradiction, suppose that $z \notin W_{a}$. Hence $z \in W_{b}$ for some $b \in V(H)$ different from $a$. Since $a$ (resp. $b$ ) is the unique vertex that minimizes the distance from $x$ (resp. $z$ ) to $H$, we infer that $d(x, a)=d(z, b)=$ $k$ and analogously that $d(y, a)=d(z, b)=k$. We claim that $a$ and $b$ are adjacent. Indeed, since $z \in W_{b}$, there must be a shortest path from $z$ to $a$, going through $b$. Since $d(z, b)=k$ 
and $d(z, a)=d(x, a)+1=k+1$, we infer $d(a, b) \leq 1$ which implies that $a$ and $b$ are adjacent.

By using the quadrangle condition for $a, x, y$, and $z$ (or, if $x$ and $y$ are adjacent, using the triangle condition for $a, x$, and $y$ ), we conclude that $x$ and $y$ have a common neighbor $t$ such that $d(a, t)=k-1$. Since $t \in I(x, a)$, clearly $t \in W_{a}$ and thus $d(b, t)=k$. Applying the quadrangle condition for $b, t, z$, and $x$, we infer that $t$ and $z$ have a common neighbor $s$ such that $d(b, s)=k-1$. It is easy to see that $t$ is not adjacent to $z$ and that $s$ is not adjacent to $x$ and $y$. Consequently, the vertices $x, y, z, s$, and $t$ induce a $K_{2,3}$ if $x$ and $y$ are not adjacent, or a $W_{4}^{-}$otherwise. This leads to a contradiction. Hence $W_{a}$ is locally convex and $\Delta$-closed, when each fiber $W_{a}$ is gated.

Now suppose that there exists an edge $u v$ with $u \in W_{a}$ and $v \in W_{b}$. Since $a$ is the gate of $u$ in $H$ and $b$ is the gate of $v$ in $H$, we conclude that $d(u, a)+d(a, b)=d(u, b) \leq$ $1+d(v, b)$ and $d(v, b)+d(b, a)=d(v, a) \leq 1+d(u, a)$. From these two inequalities, we deduce that $d(a, b)=1$.

Lemma 6. Let $a, b \in V(H)$ be two adjacent vertices. Then $U_{a b}=U_{a}$ and $U_{b a}=U_{b}$.

Proof. If $H$ has only two vertices, the assertion is trivial. Otherwise, since $H$ is a 2-connected chordal subgraph, there exists a vertex $c \in V(H)$ such that $a, b, c$ form a triangle. We first claim that $U_{a b}=U_{a c}$. Let $x \in U_{a b}$. Then there exists $y \in U_{b}$ that is adjacent to $x$ and clearly $d(a, x)=d(b, y)$. Since $c \in W_{c}$, we have $d(c, x)=d(c, y)=$ $k \geq 2$, and by the triangle condition there exists a common neighbor $z$ of $x$ and $y$ such that $d(c, z)=k-1$. It is easy to see that $z \in W_{c}$, which implies that $x \in U_{a c}$. By symmetry, we infer that $U_{a b}=U_{a c}$. Now, let $x \in U_{a}$. Then $x \in U_{a d}$ for some $d \in N(a) \cap H$. Since $H$ is 2-connected and chordal, there exists a sequence of vertices $b=c_{0}, c_{1}, \ldots, c_{m}=d$ of $H$ such that $a, c_{i}$, and $c_{i+1}$ form a triangle for all $i=0, \ldots m-1$. By the previous reasoning, this implies that $U_{a b}=U_{a c_{i}}=U_{a d}$. In particular, $x \in U_{a b}$, showing that $U_{a b}=U_{a}$.

By Lemma 5, we infer that any vertex $x \in U_{a b}=U_{a}$ has exactly one neighbor in $U_{b a}=U_{b}$. Indeed, since each fiber $W_{b}$ is gated, there cannot be a vertex not in $W_{b}$ adjacent to two vertices of $W_{b}$. This fact combined with Lemma 6 gives rise to the following natural mapping: $f_{a b}: U_{a} \longrightarrow U_{b}$ that maps $x \in U_{a}$ to the neighbor of $x$ in $U_{b}$.

Lemma 7. Let $a, b$ be two adjacent vertices of $H$. Then $U_{a}$ and $U_{b}$ are isomorphic subgraphs of $G$ and $f_{a b}$ is an isomorphism between the graphs $U_{a}$ and $U_{b}$.

Proof. Let $x, y$ be two adjacent vertices of $U_{a}$, and suppose that their neighbors $x^{\prime}, y^{\prime}$ in $U_{b}$ are not adjacent. By Lemma 5 each $W_{a}$ is gated, thus convex. Since $W_{b}$ is convex, we infer that $d_{W_{b}}\left(x^{\prime}, y^{\prime}\right)=2$. Let $z^{\prime} \in W_{b}$ be a common neighbor of $x^{\prime}$ and $y^{\prime}$. Since $d\left(y, z^{\prime}\right)=d\left(y, x^{\prime}\right)=2$, by the triangle condition we infer that there exists a common neighbor $u$ of $y, x^{\prime}$, and $z^{\prime}$. Since $W_{b}$ is $\Delta$-closed, we conclude that $u \in W_{b}$. But then $y \in U_{a}$ has two neighbors $u$ and $y^{\prime}$ in $U_{b}$, which is impossible.

Lemma 8. The subgraphs $U_{a}$ are gated for all $a \in V(H)$ and are mutually isomorphic. Their union is isomorphic to $H \square U$, where $U$ is any of $U_{a}$.

Proof. Since $H$ is connected, from Lemma 7, we immediately infer that the subgraphs $U_{a}$ are all mutually isomorphic. Since each fiber $W_{a}$ is gated, to prove that $U_{a}$ is gated it suffices to show that $U_{a}$ is locally convex and $\Delta$-closed in the subgraph $W_{a}$. 
Let $x, y \in U_{a}$ be two vertices having a common neighbor $z \in U_{a}$ and suppose that there is a vertex $s \in W_{a} \backslash U_{a}$ that is adjacent to both $x$ and $y$ but not to $z$ (the case when $s$ is adjacent to $z$ is covered by $\Delta$-closedness of $U_{a}$ established below). Let $b$ be a neighbor of $a$ in $H$ and let $x^{\prime}, z^{\prime}, y^{\prime} \in U_{b}$ be the neighbors of $x, z, y$, respectively. By Lemma 7, we conclude that $z^{\prime}$ is adjacent to $x^{\prime}$ and $y^{\prime}$ but $x^{\prime}$ and $y^{\prime}$ are not adjacent. Then $d\left(s, x^{\prime}\right)=d\left(s, y^{\prime}\right)=d\left(s, z^{\prime}\right)-1=2$ and by the quadrangle condition we find that $x^{\prime}, y^{\prime}$ and $s$ have a common neighbor $s^{\prime}$. Since $W_{b}$ is convex, $s^{\prime} \in U_{b}$ which in turn implies that $s \in U_{a}$, a contradiction. This shows that $U_{a}$ is locally convex.

Let $x, y \in U_{a}$ be two adjacent vertices and suppose that there is a vertex $s \in W_{a} \backslash U_{a}$ adjacent to both $x$ and $y$. Let $b$ be a neighbor of $a$ in $H$ and let $x^{\prime}, y^{\prime} \in U_{b}$ be the neighbors of $x, y$, respectively. By Lemma 7, we know that $x^{\prime}$ is adjacent to $y^{\prime}$. Then $d\left(s, x^{\prime}\right)=d\left(s, y^{\prime}\right)=2$ and by the triangle condition we find that $x^{\prime}, y^{\prime}$, and $s$ have a common neighbor $s^{\prime}$. Since $N(s) \subseteq U_{a}$, it implies that either $x^{\prime}$ or $y^{\prime}$ has two neighbors in $U_{a}$, a contradiction. This shows that $U_{a}$ is $\Delta$-closed. Thus $U_{a}$ is indeed gated.

The structure of the union of all $U_{a}, a \in V(H)$, is now completely described. Its vertex set is isomorphic to $V(H) \times V(U)$, where $U$ is isomorphic to $U_{a}$ for any $a \in V(H)$. For any vertices $a, c \in V(H)$ and any $x \in U_{a}, y \in U_{c}, x$ is adjacent to $y$ if and only if either $a=c$ and $x y \in E\left(U_{a}\right)$, or $a$ and $c$ are adjacent and $y$ is the unique neighbor of $x$ in $U_{c}$. Hence, the union of $U_{a}$ over all $a \in V(H)$ is isomorphic to $H \square U$.

We collected all results to conclude the proof of the implication (ii) $\Rightarrow$ (iii) of Theorem 1. We proceed by induction on the cardinality of $G$. First, if $H$ (the gated hull of $\{u, v\}$ in $G$ ) is equal to whole graph $G$, then $G$ is chordal, hence $G$ is a cage-amalgamation graph. Therefore, we can suppose that $H$ is a proper subgraph of $G$. Now, suppose that for any $a \in V(H)$, the set $W_{a}$ coincides with $U_{a}$. By Lemma $8, G$ is isomorphic to $H \square W_{a}=H \square U_{a}$, where $H$ is a chordal graph. Since $W_{a}$ has smaller cardinality than $G$ and since $W_{a}$ is a weakly modular graph without $K_{2,3}, W_{k}$, and $W_{k}^{-}, k \geq 4$ (as a gated subgraph of $G$ ), by induction hypothesis $W_{a}$ is a cage-amalgamation graph. Since Cartesian products and gated amalgams commute (see also Lemma 3.1 of [11]), $G=H \square W_{a}$ is a cageamalgamation graph as well. Finally, suppose that for some $a \in V(H)$ the set $W_{a}-U_{a}$ is nonempty. Since $U_{a}$ is gated and is a separator of $G$, we conclude that $G$ is the gated amalgam of $W_{a}$ and $G-\left(W_{a}-U_{a}\right)$ along the common gated subgraph $U_{a}$. Since both those graphs $W_{a}$ and $G-\left(W_{a}-U_{a}\right)$ have smaller cardinality that $G$, they are cageamalgamation graphs, and thus so is $G$. This concludes the proofs of the implication (ii) $\Rightarrow$ (iii) and of Theorem 1 .

\section{THE PRISM COMPLEXES OF CAGE-AMALGAMATION GRAPHS}

The second result of this article concerns the geometry of prism complexes derived from cage-amalgamation graphs. Namely, we show that all such complexes endowed with intrinsic $l_{2}$-metric are CAT(0) metric spaces. We continue with the definition of piecewise Euclidean (PE) cell complexes and of CAT(0) metric spaces.

A Euclidean cell is a convex polytope in some Euclidean space. By a PE cell complex, we will mean a space $\mathbf{X}$ formed by gluing together Euclidean cells via isometries of their faces, together with the decomposition of $\mathbf{X}$ into cells. Additionally, we assume that the intersection of two cells is either empty or a single face of each of the cells. If all cells of $\mathbf{X}$ are Euclidean simplexes (respectively, cubes), we will say that $\mathbf{X}$ is a 
simplicial (respectively, cubical) cell complex. If all cells of $\mathbf{X}$ are Euclidean prisms, that is, Cartesian products of simplices, then $\mathbf{X}$ is called a prism complex; the precise definition is given below. For a PE complex $\mathbf{X}$ denote by $V(\mathbf{X})$ and $E(\mathbf{X})$ the vertex set and the edge set of $\mathbf{X}$, namely, the set of all zero-dimensional and one-dimensional faces of $\mathbf{X}$. The pair $(V(\mathbf{X}), E(\mathbf{X}))$ is called the (underlying) graph or the 1-skeleton of $\mathbf{X}$ and is denoted by $G(\mathbf{X})$.

With each simplicial or cubical PE complex $\mathbf{X}$ one can associate in a canonical way an abstract simplicial or cubical complex. Recall that an abstract simplicial complex $\mathbf{X}$ is a collection of sets (called simplices) such that $\sigma \in \mathbf{X}$ and $\sigma^{\prime} \subseteq \sigma$ implies $\sigma^{\prime} \in \mathbf{X}$. A cubical complex $\mathbf{X}$ is a set of (graph) cubes of any dimensions that is closed under taking subcubes and nonempty intersections. Simplices or cubes of the respective complexes are called faces. The link of a vertex $x$ in a simplicial complex $\mathbf{X}$, denoted link $(x, \mathbf{X})$ is simplicial complex consisting of all simplexes $\sigma$ of $\mathbf{X}$ such that $x \notin \sigma$ and $\sigma$ together with $x$ defines a simplex of $\mathbf{X}$. A simplicial complex $\mathbf{X}$ is a flag complex (or a clique complex) if any set of vertices is included in a face of $\mathbf{X}$ whenever each pair of its vertices is contained in a face of $\mathbf{X}$ (in the theory of hypergraphs, this condition is called conformality). A flag complex can therefore be recovered by its underlying graph $G(\mathbf{X})$ : the complete subgraphs of $G(\mathbf{X})$ are exactly the simplices of $\mathbf{X}$. Conversely, for a graph $G$ one can derive a simplicial complex $\mathbf{X}(G)$ by taking all complete subgraphs (simplices) as faces of the complex. Analogously, for a graph $G$ one can also derive a cubical complex $\mathbf{C}(G)$ by taking all induced subhypercubes as faces. If $G$ is a median graph, then $\mathbf{C}(G)$ consists of all hypercubes which are obtained as Cartesian products of the prime graphs (as we noticed above, they are all two-vertex complete graphs $K_{2}$ ). The simplicial complexes arising as clique complexes of bridged graphs were characterized in [16] as simply connected simplicial complexes in which the links of vertices do not contain induced 4and 5-cycles (these complexes have been rediscovered and investigated by Januszkiewicz and Swiatkowski [26], who called them "systolic complexes" and considered them as simplicial complexes satisfying combinatorial nonpositive curvature property, see the definition below).

In the context of graphs $G$ obtained via Cartesian products and gated amalgamations from prime graphs containing cliques of arbitrary size, it is natural to associate to each prime graph $G_{i}$ of $G$ a PE simplicial complex $\mathbf{X}\left(G_{i}\right)$ obtained by replacing each simplex of the clique complex of $G_{i}$ by an Euclidean simplex. Then the prism complex of $G$ is the PE cell complex $\mathbf{H}(G)$ obtained by taking all Hamming subgraphs of $G$ (Cartesian products of complete subgraphs of prime graphs) and replacing them by respective Euclidean prisms. Then each face $\tau$ of $\mathbf{H}(G)$ is the Cartesian product $\tau=\sigma_{1} \times \cdots \times \sigma_{k}$, where $\sigma_{i}$ is a simplex of $\mathbf{X}\left(G_{i}\right), i=1, \ldots, k$. This is consistent with the standard definition of the product of two (or more) polytopes given on pp. 9-10 of the book of Ziegler [36]: given two polytopes $P \subset \mathbb{R}^{n}$ and $Q \subset \mathbb{R}^{m}$, the product of $P$ and $Q$ is the set $P \times Q=\{(x, y): x \in P, y \in Q\} . P \times Q$ is a polytope of $\operatorname{dimension} \operatorname{dim}(P)+\operatorname{dim}(Q)$, whose nonempty faces are the products of nonempty faces of $P$ and nonempty faces of $Q$. It is well known (see e.g., p. 110 of [36]) that the product $\sigma_{1} \times \cdots \times \sigma_{k}$ of Euclidean simplices $\sigma_{1}, \ldots, \sigma_{k}$ is a convex polyhedron $\tau$, called a prism. Notice that if $G$ is a median graph (or more generally, a triangle-free graph), then the prism complex of $G$ coincides with the cubical complex $\mathbf{C}(G)$ defined before.

Let $(X, d)$ be a metric space. The interval between two points $x, y$ of $X$ is the set $I(x, y)=\{z \in X: d(x, y)=d(x, z)+d(z, y)\}$; for example, in Euclidean spaces, the interval $I(x, y)$ is the closed line segment having $x$ and $y$ as its endpoints. The space 
$(X, d)$ is called Menger-convex if for any two distinct points $x, y \in X$ there exists a point $z \in I(x, y)$ different from $x, y$. A geodesic joining two points $x$ and $y$ from $X$ is the image of a (continuous) map $\gamma$ from a line segment $[0,1] \subset \mathbb{R}$ to $X$ such that $\gamma(0)=x, \gamma(1)=y$ and $d\left(\gamma(t), \gamma\left(t^{\prime}\right)\right)=\left|t-t^{\prime}\right|$ for all $t, t^{\prime} \in[0,1]$. The space $(X, d)$ is said to be geodesic if every pair of points $x, y \in X$ is joined by a geodesic (which is necessarily included in $I(x, y))$ [12]. Every complete Menger-convex metric space is geodesic [12]. Any finite PE cell complex $\mathbf{X}$ can be endowed with an intrinsic $l_{2}$-metric [12], transforming $\mathbf{X}$ into a complete geodesic space. Suppose that inside every cell of $\mathbf{X}$ the distance is measured according to the Euclidean $l_{2}$-metric. The intrinsic $l_{2}$-metric $d_{2}$ of $\mathbf{X}$ is defined by assuming that the distance between two points $x, y \in \mathbf{X}$ lying in different cells equals to the infimum of the lengths of the paths joining them. Here a path in $\mathbf{X}$ from $x$ to $y$ is a sequence $P$ of points $x=x_{0}, x_{1} \ldots x_{m-1}, x_{m}=y$ such that for each $i=0, \ldots, m-1$, there exists a cell $\tau_{i}$ containing $x_{i}$ and $x_{i+1}$; the length of $P$ is $l(P)=\sum_{i=0}^{m-1} d\left(x_{i}, x_{i+1}\right)$, where $d\left(x_{i}, x_{i+1}\right)$ is computed inside $\tau_{i}$ according to the Euclidean $l_{2}$-metric.

Now, we will briefly review the definitions and some characterizations of $\operatorname{CAT}(0)$ metric spaces (geodesic metric spaces of global nonpositive curvature). This theory originates from the classical papers of Alexandrov, Busemann, Bruhat, Cartan, Hadamard, Tits, Toponogov, and others. In most generality it has been defined in the seminal paper of Gromov [22]. CAT(0) spaces represent a far-reaching common generalization of Euclidean spaces, classical real-hyperbolic spaces (which are spaces with negative curvature), and Riemannian manifolds of strictly negative sectional curvature. CAT(0) spaces play a vital role in modern combinatorial group theory, where various versions of hyperbolicity are related to group-theoretic properties [21], [22]; many arguments in this area have a strong metric graph-theoretic flavor. For a survey in more depth and background, the reader should refer to the book of Bridson and Haefliger [12], whose terminology we follow.

A geodesic triangle $\Delta\left(x_{1}, x_{2}, x_{3}\right)$ in a geodesic metric space $(X, d)$ consists of three distinct points in $X$ (the vertices of $\Delta$ ) and a geodesic between each pair of vertices (the sides of $\Delta)$. A comparison triangle for $\Delta\left(x_{1}, x_{2}, x_{3}\right)$ is a triangle $\Delta\left(x_{1}^{\prime}, x_{2}^{\prime}, x_{3}^{\prime}\right)$ in the Euclidean plane $\mathbb{E}^{2}$ such that $d_{\mathbb{E}^{2}}\left(x_{i}^{\prime}, x_{j}^{\prime}\right)=d\left(x_{i}, x_{j}\right)$ for $i, j \in\{1,2,3\}$. A geodesic metric space $(X, d)$ is defined to be a $C A T(O)$ space [22] if all geodesic triangles $\Delta\left(x_{1}, x_{2}, x_{3}\right)$ of $X$ satisfy the comparison axiom of Cartan-Alexandrov-Toponogov (this explains the acronym $\mathrm{CAT}(0))$ :

If $y$ is a point on the geodesic between $x_{1}$ and $x_{2}$ in the triangle $\Delta\left(x_{1}, x_{2}, x_{3}\right)$ and $y^{\prime}$ is the unique point on the line segment $\left[x_{1}^{\prime}, x_{2}^{\prime}\right]$ of the comparison triangle $\Delta\left(x_{1}^{\prime}, x_{2}^{\prime}, x_{3}^{\prime}\right)$ such that $d_{\mathbb{E}^{2}}\left(x_{i}^{\prime}, y^{\prime}\right)=d\left(x_{i}, y\right)$ for $i=1,2$, then $d\left(x_{3}, y\right) \leq d_{\mathbb{E}^{2}}\left(x_{3}^{\prime}, y^{\prime}\right)$.

This simple axiom turns out to be very powerful, because CAT(0) spaces have many properties and can be characterized in several natural ways. For example, a geodesic metric space $(X, d)$ is $\operatorname{CAT}(0)$ if and only if the distance function $f(t)=d(\alpha(t), \beta(t))$ is convex for any geodesics $\alpha$ and $\beta$. From this property, one can immediately conclude that in CAT( 0$)$ spaces (such as in bridged graphs) the $r$-neighborhoods $B_{r}(A)=\{x$ : $d(x, A) \leq r\}$ of convex sets $A$ are convex. The convexity of balls yields that CAT $(0)$ spaces are contractible. The convexity of the distance function also implies that CAT $(0)$ spaces do not contain isometrically embedded cycles. Any two points of a CAT(0) space can be joined by a unique geodesic; furthermore, a PE complex $\mathbf{X}$ is CAT(0) if and only if any two points of $\mathbf{X}$ can be joined in $\mathbf{X}$ by a unique geodesic. Other characterizations (in particular, via links) and properties of CAT(0) PE complexes can be found in the 
book [12]. In the case of cube complexes, Gromov [22] presented a nice combinatorial characterization of CAT( 0$)$ condition: a cube complex $\mathbf{X}$ is CAT(0) if and only if $\mathbf{X}$ is simply connected and the links of vertices are flag simplicial complexes.

Now, we formulate the second result of this article.

Theorem 2. If $G$ is a cage-amalgamation graph, then any prism complex $\mathbf{H}(G)$ derived from $G$ and equipped with the intrinsic $l_{2}$-metric $d_{2}$ is a $C A T(0)$ metric space.

It was already noticed in [16], [23] that clique complexes of chordal graphs lead to CAT(0) simplicial complexes. Gromov called them (p. 121 of [23]) tree-like polyhedra and also noticed to be CAT( 0$)$. In the rest of this section, we prove Theorem 2.

The proof of Theorem 2 uses the decomposition scheme from Theorem 1 and runs in three steps: first we show that a PE simplicial complex derived from the clique complex of a chordal graph is CAT(0), then we establish that the prism complex of a Cartesian product of chordal graphs is $\mathrm{CAT}(0)$ as well, and finally we show that gated amalgams of cage-amalgamation graphs preserve the $\mathrm{CAT}(0)$ property of their prism complexes. The proof employs the following known property of CAT(0) spaces due to Reshetnyak and which is a particular case of the basic gluing theorem 11.1 of [12].

Gluing Theorem. If $\left(X_{1}, d_{1}\right)$ and $\left(X_{2}, d_{2}\right)$ are two CAT( 0$)$ spaces, $A_{i}$ is a convex nonempty subset of $\left(X_{i}, d_{i}\right), i=1,2$, and there exists an isometry $\varphi$ between $A_{1}$ and $A_{2}$, then the metric space $\left(X_{1} \cup X_{2}, d\right)$ obtained by gluing $X_{1}$ and $X_{2}$ along the sets $A_{1}$ and $A_{2}$ is $C A T(0)$.

The metric space $\left(X_{1} \cup X_{2}, d\right)$ is obtained by identifying $A_{1}$ and $A_{2}$ according to $\varphi$ and $d$ is defined to be $d_{1}$ on $X_{1}, d_{2}$ on $X_{2}$, and $d(x, y)=\inf \left\{d_{1}(x, a)+d_{2}(a, y): a \in A_{2}=\right.$ $\left.\varphi\left(A_{1}\right)\right\}$ if $x \in A_{1}$ and $y \in A_{2}$.

We first recall the proof of Corollary 8.4 of [16] showing that any simplicial complex $\mathbf{X}(G)$ derived from the clique complex of a finite chordal graph $G$ is CAT $(0)$. We recall this short proof here because the proof of Theorem 2 is based on the same principle. We proceed by induction on the number of vertices of $G$. Let $x$ be a simplicial vertex of $G$. Then $x$ belongs to the unique maximal by inclusion simplex $\sigma$ of $\mathbf{X}(G)$ induced by $x$ and all its neighbors in $G$. Consequently, $\mathbf{X}(G)$ can be obtained by gluing $\sigma$ and $\mathbf{X}^{\prime}$, where $\mathbf{X}^{\prime}$ is the subcomplex of $\mathbf{X}(\mathbf{G})$ spanned by the facet $\sigma^{\prime}$ of $\sigma$ not containing $x$ (i.e., by $\operatorname{link}(x, \mathbf{X}(\mathbf{G}))$ and the maximal simplexes of $\mathbf{X}(G)$ distinct from $\sigma$ (in fact, $\mathbf{X}^{\prime}$ is a simplicial complex derived from the clique complex of the chordal graph $G^{\prime}:=G-\{x\}$ ). Since the gluing is performed along a convex set $\sigma^{\prime}$ of both complexes $\sigma$ and $\mathbf{X}^{\prime}$, from the result of Reshetnyak mentioned above, we obtain that $\mathbf{X}(G)$ is $\operatorname{CAT}(0)$ if and only if $\sigma$ and $\mathbf{X}^{\prime}$ are $\operatorname{CAT}(0)$. Since $\mathbf{X}^{\prime}=\mathbf{X}\left(G^{\prime}\right)$ and the graph $G^{\prime}$ is chordal, by the induction assumption, $\mathbf{X}^{\prime}$ is $\mathrm{CAT}(0)$, and we are done. In view of perfect elimination schemes of chordal graphs $G, \mathbf{X}(G)$ can be written as a directed union $\bigcup_{i=1}^{n} \mathbf{X}_{i}$ where $\mathbf{X}_{i}=\mathbf{X}_{i-1} \cup \sigma_{i}$ and the simplex $\sigma_{i}$ meets $\mathbf{X}_{i-1}$ over a single face $\sigma_{i}^{\prime}$.

Now suppose that $G$ is a cage-amalgamation graph whose prime graphs are the chordal graphs $G_{1}, \ldots, G_{m}$. Each of these graphs occurs as a gated subgraph of $G$. Let $x$ be a simplicial vertex of $G_{1}$. Denote by $\sigma_{x}$ the unique maximal by inclusion simplex of $\mathbf{X}\left(G_{1}\right)$ induced by $x$ and all its neighbors in $G_{1}$ and let $\sigma_{x}^{\prime}$ be the facet of $\sigma_{x}$ not containing the vertex $x$. For each vertex $a$ of $G_{1}$, denote by $W_{a}$ its fiber in $G$ relative to some copy of the gated subgraph $G_{1}$. From Lemma 5 , each such fiber $W_{a}$ is gated. From Lemmas 7 and 8 , we conclude that the boundaries $U_{a}$ of these fibers $W_{a}$ are isomorphic gated subgraphs of $G$. Denote by $\mathbf{H}_{\sigma_{x}}$ (resp. $\mathbf{H}_{\sigma_{x}^{\prime}}$ ) the prism complexes of the subgraphs of $G$ induced by the 


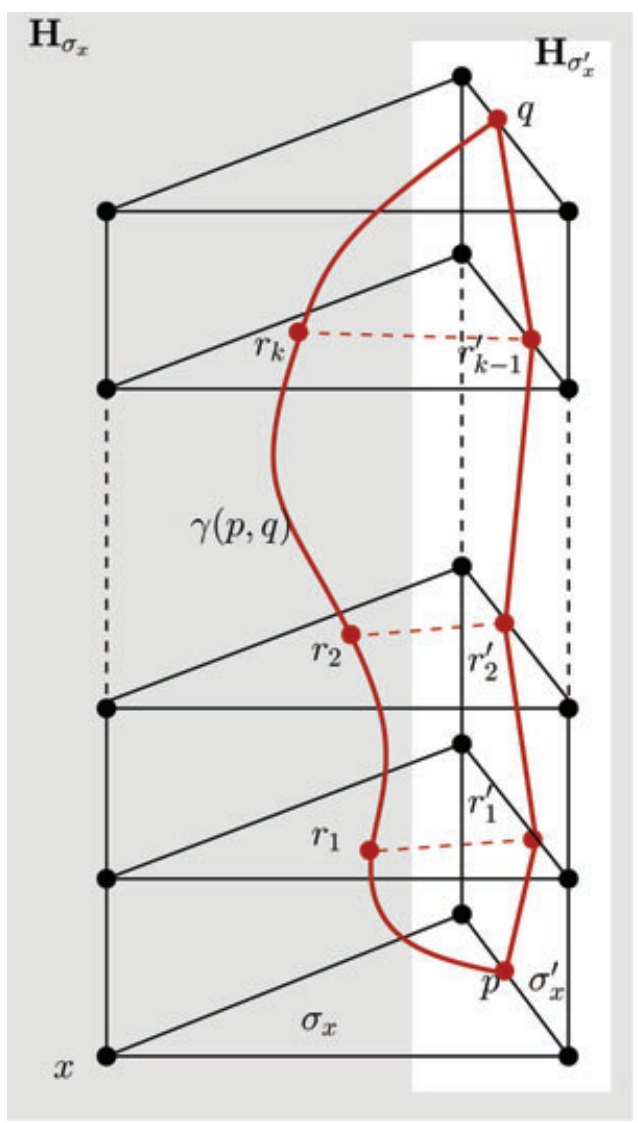

FIGURE 6. To the proof of Lemma 9.

unions $\bigcup_{a \in \sigma_{x}} U_{a}$ (resp. $\bigcup_{a \in \sigma_{x}^{\prime}} U_{a}$ ). Notice that $\mathbf{H}_{\sigma_{x}^{\prime}}$ is a subcomplex of $\mathbf{H}_{\sigma_{x}}$ and that both $\mathbf{H}_{\sigma_{x}^{\prime}}$ and $\mathbf{H}_{\sigma_{x}}$ are subcomplexes of $\mathbf{H}(G)$.

Lemma 9. If $p, q$ are two points of $\mathbf{H}_{\sigma_{x}^{\prime}}$, then any geodesic connecting $p$ and $q$ in $\mathbf{H}_{\sigma_{x}}$ is contained in $\mathbf{H}_{\sigma_{x}^{\prime}}$.

Proof. Suppose by way of contradiction that such a geodesic $\gamma(p, q)$ contains a point in the set $\mathbf{H}_{\sigma_{x}}-\mathbf{H}_{\sigma_{x}^{\prime}}$ (see Fig. 6 for an illustration). Let $\pi_{1}, \ldots, \pi_{k}$ be the maximal by inclusion prisms of $\mathbf{H}_{\sigma_{x}}$ intersected by $\gamma(p, q)$ labeled in order in which they are traversed by $\gamma(p, q)$. Let $\pi_{i}^{\prime}$ be the facet of $\pi_{i}$ in $\mathbf{H}_{\sigma_{x}^{\prime}}$, that is, $\pi_{i}^{\prime}=\pi_{i} \cap \mathbf{H}_{\sigma_{x}^{\prime}}$. The intersection of any two consecutive prisms $\pi_{i}$ and $\pi_{i+1}$ is a face $\tau_{i}$ of each of them. Let $\tau_{i}^{\prime}$ denote the facet of $\tau_{i}$ in $\pi_{i}^{\prime}$ (and $\pi_{i+1}^{\prime}$ ). Let $r_{i} \in \gamma(p, q) \cap \tau_{i}$. The orthogonal projection of each prism $\pi_{i}$ on its facet $\pi_{i}^{\prime}$ is a nonexpansive map $f_{i}$. Moreover, each point $r_{i}$ is mapped by $f_{i}$ and $f_{i+1}$ to the same point $r_{i}^{\prime}$ belonging to $\tau_{i}^{\prime}$. As a result, the length of the path $\gamma^{\prime}(p, q)$ between $p=r_{0}^{\prime}$ and $q=r_{k}^{\prime}$ consisting of line segments connecting the consecutive points $p, r_{1}^{\prime}, r_{2}^{\prime}, \ldots, r_{k-1}^{\prime}, q$ is at most the length of $\gamma(p, q)$. Since $p, q \in \mathbf{H}_{\sigma_{x}^{\prime}}$ and $\gamma(p, q)$ passes via a point of $\mathbf{H}_{\sigma_{x}}-\mathbf{H}_{\sigma_{x}^{\prime}}$, at least one of the orthogonal projections $r_{i}^{\prime} r_{i+1}^{\prime}$ must be strictly smaller than the length of $\gamma\left(r_{i}, r_{i+1}\right)$ (the portion of $\gamma(p, q)$ comprised between $r_{i}$ 
and $\left.r_{i+1}\right)$, thus $\gamma^{\prime}(p, q)$ is strictly shorter than $\gamma(p, q)$, completing the proof of the lemma.

Now, by induction on the number of vertices of $G$, we will establish that if $G$ is a Cartesian product of chordal graphs $G_{1}, \ldots, G_{m}$, then $\mathbf{H}(G)$ is CAT(0). This is obviously true if each $G_{i}$ is a clique. So, suppose without loss of generality that $G_{1}$ is not a clique. Let $x$ be a simplicial vertex of $G_{1}$. From Lemma 9, we know that the subcomplex $\mathbf{H}_{\sigma_{x}^{\prime}}$ is convex (with respect to the $d_{2}$-metric) in $\mathbf{H}_{\sigma_{x}}$. Let $G_{1}^{\prime}:=G_{1}-\{x\}$ and $G^{\prime}:=G_{1}^{\prime} \square G_{2} \square \ldots \square G_{m}$. By induction assumption, $\mathbf{H}_{\sigma_{x}}$ and $\mathbf{H}\left(G^{\prime}\right)$ are CAT(0) spaces. Since $\mathbf{H}(G)$ is obtained by gluing $\mathbf{H}_{\sigma_{x}}$ and $\mathbf{H}\left(G^{\prime}\right)$ along $\mathbf{H}_{\sigma_{x}^{\prime}}$ and $\mathbf{H}_{\sigma_{x}^{\prime}}$ is convex in $\mathbf{H}_{\sigma_{x}}$, to apply the basic gluing theorem, it suffices to show that $\mathbf{H}_{\sigma_{x}^{\prime}}$ is convex in $\mathbf{H}\left(G^{\prime}\right)$. This is obviously true when $G_{1}^{\prime}=\sigma_{x}^{\prime}$. Otherwise, $G_{1}^{\prime}$ contains a simplicial vertex $y \notin \sigma_{x}^{\prime}$. Let $G^{\prime \prime}=G_{1}^{\prime}-\{y\}$ and assume by induction assumption that $\mathbf{H}_{\sigma_{x}^{\prime}}$ is convex in $\mathbf{H}\left(G^{\prime \prime}\right)$. Therefore, if $\mathbf{H}_{\sigma_{x}^{\prime}}$ is not convex in $\mathbf{H}\left(G_{1}^{\prime}\right)$, then we can find two points $p, q \in \mathbf{H}_{\sigma_{x}^{\prime}}$ and a geodesic $\gamma(p, q)$ between $p$ and $q$ in $\mathbf{H}\left(G_{1}^{\prime}\right)$ containing at least one point $z \in \mathbf{H}\left(G_{1}^{\prime}\right)-\mathbf{H}\left(G^{\prime \prime}\right)=\mathbf{H}_{\sigma_{y}}-\mathbf{H}_{\sigma_{y}^{\prime}}$. Then $\gamma(p, q)$ contains two points $p^{\prime}, q^{\prime} \in \mathbf{H}_{\sigma_{y}^{\prime}}$ such that $z$ belongs to the portion $\gamma\left(p^{\prime}, q^{\prime}\right)$ of $\gamma(p, q)$ comprised between $p^{\prime}$ and $q^{\prime}$. Since $\gamma(p, q)$ is a geodesic, necessarily $\gamma\left(p^{\prime}, q^{\prime}\right)$ is a geodesic between $p^{\prime}$ and $q^{\prime}$. This however contradicts the convexity of $\mathbf{H}_{\sigma_{y}^{\prime}}$ in $\mathbf{H}_{\sigma_{y}}$ established in Lemma 9. This shows that $\mathbf{H}_{\sigma_{x}^{\prime}}$ is convex in $\mathbf{H}\left(G^{\prime}\right)$ as well, and therefore we can apply the gluing theorem.

Finally, suppose that a graph $G$ is a gated amalgam of two cage-amalgamation graphs $G^{\prime}$ and $G^{\prime \prime}$ along a gated subgraph $G_{0}$. Suppose by induction assumption that $\mathbf{H}\left(G^{\prime}\right)$ and $\mathbf{H}\left(G^{\prime \prime}\right)$ are CAT $(0)$ spaces. To use the gluing theorem again, it suffices to show that $\mathbf{H}\left(G_{0}\right)$ is convex (with respect to the intrinsic $d_{2}$-metric) in both $\mathbf{H}\left(G^{\prime}\right)$ and $\mathbf{H}\left(G^{\prime \prime}\right)$, say in $\mathbf{H}\left(G^{\prime}\right)$.

Lemma 10. If $G_{0}$ is a gated subgraph of a cage-amalgamation graph $G^{\prime}$, then $\mathbf{H}\left(G_{0}\right)$ is convex in $\mathbf{H}\left(G^{\prime}\right)$.

Proof. We proceed by induction on the number of vertices of $G^{\prime}$. Since $G_{0}$ is different from $G^{\prime}$, there exists a vertex $y$ of $G_{0}$ that has a neighbor $y^{\prime} \in V\left(G^{\prime}\right) \backslash V\left(G_{0}\right)$. Let $H$ be the gated hull of the edge $y y^{\prime}$. Consider the partition of $G^{\prime}$ into fibers $W_{a}$ with respect to the vertices $a$ of $H$. Clearly, the gated subgraph $G_{0}$ is completely contained in the fiber $W_{y}$ of $y$. By Proposition 2, $H$ is either a 2-connected chordal graph or an edge. In both cases, $H$ contains a simplicial vertex $x$ different from $y$. Denote by $\sigma_{x}$ the simplex defined by the unique maximal complete subgraph of $H$ containing $x$ and let $\sigma_{x}^{\prime}$ be the facet of $\sigma_{x}$ not containing the vertex $x$. Let $D$ be the subgraph of $G^{\prime}$ induced by all vertices not belonging to the fiber $W_{x}$. Since $x$ is a simplicial vertex of $H$, it can be easily seen that $D$ is an isometric (in fact a convex) subgraph of $G^{\prime}$. D is a cage-amalgamation graph: its prime graphs are the same as those of $G^{\prime}$ with the single exception that $H$ is replaced by $H-\{x\}$. Moreover, $G_{0}$ is a gated subgraph of $D$. Thus, by induction assumption, we can suppose that $\mathbf{H}\left(G_{0}\right)$ is a convex subcomplex of $\mathbf{H}(D)$. Now, suppose by way of contradiction that $\mathbf{H}\left(G_{0}\right)$ is not convex in $\mathbf{H}\left(G^{\prime}\right)$. Then there exist two points $p, q \in \mathbf{H}\left(G_{0}\right)$ such that the geodesic $\gamma(p, q)$ connecting $p$ and $q$ in $\mathbf{H}\left(G^{\prime}\right)$ does not belong to $\mathbf{H}\left(G_{0}\right)$ (see Fig. 7 for an illustration). Since $\mathbf{H}\left(G_{0}\right)$ is convex in $\mathbf{H}(D), \gamma(p, q)$ contains at least one point $z$ not belonging to $\mathbf{H}(D)$. Then $\gamma(p, q)$ necessarily contains two points $p^{\prime}, q^{\prime} \in \mathbf{H}_{\sigma_{x}^{\prime}}$ (where $\mathbf{H}_{\sigma_{x}^{\prime}}$ is defined as before) such that $z$ belongs to the part $\gamma\left(p^{\prime}, q^{\prime}\right)$ of $\gamma(p, q)$ comprised between the points $p^{\prime}$ and $q^{\prime}$. Since $\gamma\left(p^{\prime}, q^{\prime}\right)$ is a part of a geodesic, $\gamma\left(p^{\prime}, q^{\prime}\right)$ is a geodesic itself. If $\gamma\left(p^{\prime}, q^{\prime}\right)$ (and therefore $z$ ) is contained in the subcomplex $\mathbf{H}_{\sigma_{x}}$ of $\mathbf{H}\left(W_{x}\right)$, then 


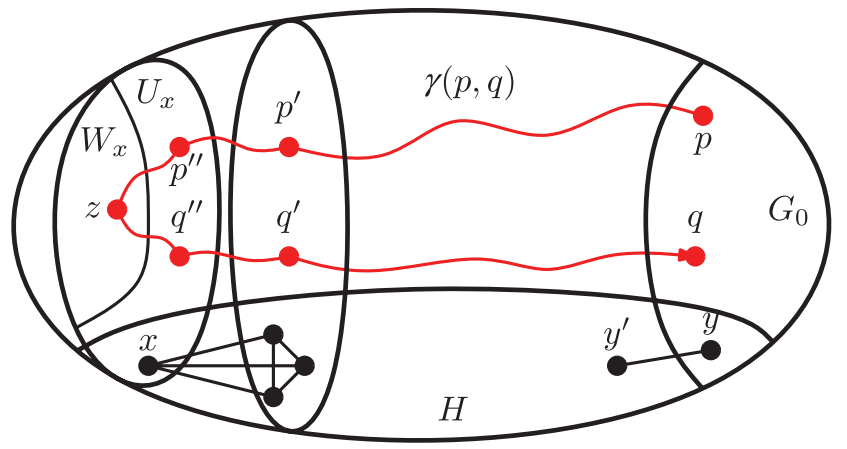

FIGURE 7. To the proof of Lemma 10.

we obtain a contradiction with Lemma 9 asserting the convexity of $\mathbf{H}_{\sigma_{x}^{\prime}}$ in $\mathbf{H}_{\sigma_{x}}$. Thus, we can suppose that $\gamma\left(p^{\prime}, q^{\prime}\right)$ contains some points (say $z$ itself) in $\mathbf{H}\left(W_{x}\right)-\mathbf{H}_{\sigma_{x}}$. Then necessarily $\gamma\left(p^{\prime}, q^{\prime}\right)$ contains two points $p^{\prime \prime}, q^{\prime \prime} \in \mathbf{H}\left(U_{x}\right)$ such that $z$ belongs to the portion $\gamma\left(p^{\prime \prime}, q^{\prime \prime}\right)$ between $p^{\prime \prime}$ and $q^{\prime \prime}$. Again, $\gamma\left(p^{\prime \prime}, q^{\prime \prime}\right)$ is a geodesic as a part of a larger geodesic. But this means that $\mathbf{H}\left(U_{x}\right)$ is not a convex subcomplex of $\mathbf{H}\left(W_{x}\right)$, contrary to the fact that $U_{x}$ is a gated subgraph of a cage-amalgamation graph $W_{x}$ having less vertices than the graph $G^{\prime}$. This contradiction establishes Lemma 10 .

From Lemma 10 we conclude that $\mathbf{H}\left(G_{0}\right)$ is convex in $\mathbf{H}\left(G^{\prime}\right)$ and $\mathbf{H}\left(G^{\prime \prime}\right)$, therefore the gated amalgamation of $G^{\prime}$ and $G^{\prime \prime}$ along $G_{0}$ translates into gluing two CAT(0) spaces $\mathbf{H}\left(G^{\prime}\right)$ and $\mathbf{H}\left(G^{\prime \prime}\right)$ along a convex subspace $\mathbf{H}\left(G_{0}\right)$, thus $\mathbf{H}(G)$ is CAT(0) by the gluing theorem. This concludes the proof of Theorem 2 .

We conclude the article with two open questions.

Question 1. Is it true that the graphs $G$ which can be obtained by successive gated amalgams from Cartesian products of bridged graphs are exactly the weakly modular graphs not containing $K_{2,3}$, the wheels $W_{4}$ and $W_{5}$, and the almost-wheels $W_{k}^{-}$for $k \geq 4$ ?

Question 2. Characterize the triangle-square complexes (i.e., the two-dimensional complexes obtained by taking all graph triangles $C_{3}$ and squares $C_{4}$ as faces) of cageamalgamation graphs and, more generally, of graphs obtained by gated amalgams from Cartesian products of bridged graphs (i.e., graphs from Question 1)? In particular, is it true that those complexes are exactly the simply connected triangle-square complexes whose underlying graphs do not contain $K_{2,3}$, the wheels $W_{k}$, and the almost-wheels $W_{k}^{-}$for $k \geq 4$ in the cage-amalgamation case and the simply connected triangle-square complexes whose underlying graphs do not contain $K_{2,3}$, the wheels $W_{4}, W_{5}$, and the almost-wheels $W_{k}^{-}$for $k \geq 4$ in the second case? In other words, is it possible to replace the global metric condition of "weak modularity" by a topological condition of "simple connectivity"?

\section{ACKNOWLEDGMENTS}

This research was supported in part by the French-Slovenian Egide PROTEUS project "Distances, structure and products of graphs." The first and the fourth author are also with 
the Institute of Mathematics, Physics and Mechanics, Jadranska 19, 1000 Ljubljana, and were also supported by the Ministry of Science and Technology of Slovenia under the grants J1-2043 and P1-0297. The third author was also supported by the TEOMATRO grant ANR-10-BLAN 0207.

\section{REFERENCES}

[1] H.-J. Bandelt, Retracts of hypercubes, J Graph Theory 8 (1984), 501-510.

[2] H.-J. Bandelt and V. Chepoi, A Helly theorem in weakly modular space, Discrete Math 126 (1996), 25-39.

[3] H.-J. Bandelt and V. Chepoi, Decomposition and $l_{1}$-embedding of weakly median graphs, Eur J Combin 21 (2000), 701-714.

[4] H.-J. Bandelt and V. Chepoi, The algebra of metric betweenness I: Subdirect representation and retracts, Eur J Combin 28 (2007), 1640-1661.

[5] H.-J. Bandelt and V. Chepoi, The algebra of metric betweenness II: Axiomatics of weakly median graphs, Eur J Combin 29 (2008), 676-700.

[6] H.-J. Bandelt and V. Chepoi, Metric graph theory and geometry: A survey, In: Surveys on Discrete and Computational Geometry: Twenty Years Later (J. E. Goodman, J. Pach, and R. Pollack, Eds.), Contemp Math 453, AMS, Providence, RI, (2008), 49-86.

[7] H.-J. Bandelt and J. Hedlíková, Median algebras, Discrete Math 45 (1983), $1-30$.

[8] H.-J. Bandelt, H. M. Mulder, and E. Wilkeit, Quasi-median graphs and algebras, J Graph Theory 18 (1994), 681-703.

[9] B. Brešar, Arboreal structure and regular graphs of median-like classes, Discuss Math Graph Theory 23 (2003), 215-225.

[10] B. Brešar, S. Klavžar, and R. Škrekovski, Quasi-median graphs, their generalizations, and tree-like equalities, Eur J Combin 24 (2003), 557-572.

[11] B. Brešar and A. Tepeh Horvat, Cage-amalgamation graphs, a common generalization of chordal and median graphs, Eur J Combin 30 (2009), 1071-1081.

[12] M. Bridson and A. Haefliger, Metric Spaces of Non-Positive Curvature, Springer, Berlin, 1999.

[13] M. Chastand, Fiber-complemented graphs I: Structure and invariant subgraphs, Discrete Math 226 (2001), 107-141.

[14] M. Chastand, Fiber-complemented graphs II: Retractions and endomorphisms, Discrete Math 268 (2003), 81-101.

[15] V. Chepoi, Classifying graphs by means of metric triangles, Metody Diskret Anal 49 (1989), 75-93 (in Russian).

[16] V. Chepoi, Graphs of some CAT(0) complexes, Adv Appl Math 24 (2000), 125-179.

[17] M. Farber, Bridged graphs and geodesic convexity, Discrete Math 66 (1987), 249-257.

[18] M. Farber and R. E. Jamison, On local convexity in graphs, Discrete Math 66 (1987), 231-247. 
[19] T. Feder, Stable networks and product graphs, Mem Am Math Soc 555 (1995).

[20] M. C. Golumbic, Algorithmic Graph Theory and Perfect Graphs, Academic Press, New York, 1980.

[21] E. Ghys and P. de la Harpe (Eds.), Les Groupes Hyperboliques d'après M. Gromov, Birkhäuser, Progress in Mathematics, 83, 1990.

[22] M. Gromov, Hyperbolic groups, In: Essays in Group Theory, S M. Gersten (ed.), MSRI Publ., vol 8, Springer, New York, 1987, pp. 75-263.

[23] M. Gromov, CAT $(\kappa)$-spaces: Construction and concentration, Zapiski Nauchnykh Seminarov POMI 280 (2001), 101-140.

[24] W. Imrich and S. Klavžar, Product Graphs: Structure and Recognition, WileyInterscience Publication, New York, 2000.

[25] J. R. Isbell, Median algebra, Trans Am Math Soc 260 (1980), 319-362.

[26] T. Januszkiewicz and J. Swiatkowski, Simplicial nonpositive curvature, Publ Math IHES 104 (2006), 1-85.

[27] H. M. Mulder, The structure of median graphs, Discrete Math 24 (1978), 197-204.

[28] H. M. Mulder, The Interval Function of a Graph, 132, Math. Centre Tracts, Amsterdam, 1980.

[29] M. Roller, Poc sets, median algebras and group actions, preprint, University of Southampton, UK, 1998.

[30] D. Rose, R. Tarjan, and G. Lueker, Algorithmic aspects of vertex elimination on graphs, SIAM J Comput 5 (1976), 266-283.

[31] V. Soltan and V. Chepoi, Conditions for invariance of set diameter under $d$-convexification in a graph, Cybernetics 6 (1983), 14-18.

[32] R. E. Tarjan and M. Yannakakis, Simple linear-time algorithms to test chordality of graphs, test acyclicity of hypergraphs, and selectively reduce acyclic hypergraphs, SIAM J Comput 13 (1984), 566-579.

[33] M. van de Vel, Matching binary convexities, Topol Appl 16 (1983), 207-235.

[34] M. van de Vel, Theory of Convex Structures, Elsevier Science Publishers, Amsterdam, 1993.

[35] E. Wilkeit, The retracts of Hamming graphs, Discrete Math 102 (1992), 197218.

[36] G. M. Ziegler, Lectures on Polytopes, Springer-Verlag, New York, 1995. 